\title{
ROTULAGEM DE SALGADINHOS A BASE DE MILHO: ANÁLISE DA ADEQUAÇÃO AS LEGISLAÇÕES VIGENTES
}

\section{LABELING OF MAIZE-BASED SALGADINHANS: ANALYSIS OF FITNESS APPLICABLE LEGISLATION}

\author{
Mychelle de Lira Andrade ${ }^{1}$; Jeniffer Viviany dos Santos Fonseca ${ }^{2}$; João Vitor Fonseca \\ Feitoza ${ }^{3}$ Lidia Paloma da Silva Nogueira ${ }^{4}$; Gerbson Vicente de Andrade Silva ${ }^{5}$
}

DOI: https://doi.org/10.31692/978-65-991061-7-0.53-64

\section{RESUMO}

A rotulagem de alimentos tem como objetivo principal a comunicação entre o consumidor e o produto, no entanto, há uma necessidade de fiscalizar os alimentos quanto às informação contidas nos rótulos, confrontando com as legislações vigentes que exigem a conformidade dos rótulos. A Resolução da Diretoria Colegiada (RDC) n 259 de 2002, e a n 359 e 360 de 2003, estabelecem as principais resoluções referentes à rotulagem dos alimentos industrializados. Os salgadinhos são os alimentos industrializados de maior preferência, como também de consumo, principalmente por crianças. Por isso, com base na importância da conformidade da rotulagem para a orientação do consumidor, objetivou-se com esse trabalho verificar a rotulagem de diferentes marcas de salgadinhos a base de milho comercializados na cidade de Pombal, Paraíba, em comparação com as legislações vigentes. A pesquisa foi realizada com 7 diferentes marcas de salgadinhos a base de milho comercializadas em supermercados, mercadinhos e lanchonetes. As informações contidas nos rótulos foram coletadas e analisadas através de uma lista de verificação, adaptada, com 36 itens de múltipla escolha, disponibilizada pela Agência Nacional de Vigilância Sanitária (ANVISA) na internet, tendo como base o Decreto-lei n 989 de 19691, RDC n ${ }^{\circ} 259$ de 2002, RDC n 344 de 2002, RDC $n^{\circ} 360$ de 2003, RDC $n^{\circ} 123$ de 2004 e a Lei $\mathbf{n}^{\circ} \mathbf{1 0 . 6 7 4}$ de 2003. A maioria das marcas apresentaram elevada conformidade, estando elas com valores variando de 30,55 a $61,11 \%$. Na categoria de Designação de produtos o percentual de conformidades foi superior aos de não conformidades e não se aplicam, expressando valores de 57,14, 28,57 e 14,28\% respectivamente. Conclui-se que as marcas atendem a maioria dos iten avaliados, tendo em vista que mais de $90 \%$ estavam em conformidade com as legislações vigentes.

Palavras-Chave: Alimento infantil, Industrializados, Informação nutricional, RDC.

\begin{abstract}
Food labeling has as main objective the communication between the consumer and the product, however, there is a need to inspect the food for the information contained in the labels, confronting with the current legislation that require the conformity of the labels. The Resolution of the Collegiate Board of Directors (RCBD) $n^{\circ} 259$ of 2002, and $n^{\circ} 359$ and 360 of 2003, establish the main resolutions regarding the labeling of industrialized foods. Snacks are the most industrialized foods, as well as consumer foods, especially for children. Therefore, based on the importance of the conformity of the labeling to the orientation of the consumer, this work aimed to verify the labeling of different brands of corn-based snacks marketed in the city of Pombal, Paraíba, in comparison with the current legislation. The

\footnotetext{
${ }^{1}$ Pós-Graduanda em Ciência e Tecnologia de Alimentos, UFPB, mychelle xd@ hotmail.com

2 Pós-Graduanda em Ciência e Tecnologia de Alimentos, UFPB, viviany 97@ hotmail.com

${ }^{3}$ Pós-Graduando em Tecnologia Agroalimentar, UFPB, joaovitorlg95@ hotmail.com

${ }^{4}$ Pós-Graduanda lato sensu em Ciência e Tecnologia de Alimentos, IFRN, lidiapaloma28@gmail.com

${ }^{5}$ Engenheiro de Alimentos, Universidade Federal de Campina Grande, gerbsonandrade@gmail.com
} 
research was carried out with 7 different brands of corn-based snack foods marketed in supermarkets, grocery stores and snack bars. The information contained in the labels was collected and analyzed through a checklist, adapted with 36 multiple choice items, made available by the National Health Surveillance Agency (NHSA) on the internet, based on Decree-Law n 989 of 1969, RCBD n 259 of 2002, RCBD n 344 of 2002, RCBD n 360 of 2003, RCBD $n^{\circ} 123$ of 2004 and Law $n^{\circ} 10.674$ of 2003. Most brands showed high compliance, with values varying from 30,55 to $61,11 \%$. In the Product Designation category, the percentage of conformities was higher than those of nonconformities and did not apply, expressing values of $57,14,28,57$ and $14,28 \%$, respectively. It is concluded that the brands meet most of the iten evaluated, considering that more than $90 \%$ were in compliance with the laws in force.

Keywords: Infant food, Industrialized, Nutrition information, RCBD.

\section{INTRODUÇÃO}

$\mathrm{O}$ rótulo presente nos alimentos industrializados tem como principal objetivo a comunicação entre o consumidor e o produto. Essas informações nutricionais servem de orientação para as pessoas, sobre o que elas estão consumindo, porém devido à falta de conhecimento sobre como utiliza-las os consumidores acabam não atribuindo importância a essas informações (BENDINO et al., 2012).

As Resoluções da Diretoria Colegiada (RDC) n 259, de 2002, n 359 e 360, de 2003, estabelecem as principais resoluções referentes à rotulagem dos alimentos industrializados, pois determinam os regulamentos técnicos para a rotulagem de alimentos embalados, o tipo de porção por grupos de alimentos e a rotulagem nutricional, respectivamente. Essa estrutura normativa é uma ferramenta de bastante importância, pois disponibiliza de forma obrigatória e adequada as informações que devem conter nos rótulos dos produtos disponíveis no mercado (CÂMARA et al.,2008).

Os salgadinhos são alimentos industrializados que são processados com alta concentração de sal e lipídios, também possuem um alto teor de carboidratos. Eles apresentam uma textura altamente apreciada pelos consumidores, especialmente pelas crianças, por serem produzidos a partir de uma mistura de milho ou trigo, além de serem aromatizados e salgados, tornando-os mais aceitáveis ao paladar (PEIXOTO et al., 2014).

O Guia Alimentar para a População Brasileira (2006) recomenda o consumo de salgadinhos no máximo uma vez por semana. De acordo com a legislação brasileira, produtores, indústrias e empresas que comercializam alimentos devem garantir a qualidade dos produtos e proteger a saúde dos consumidores. Com base nisso a rotulagem de alimentos é de fundamental importância para a segurança do consumidor, uma vez que as informações presentes no rotulo evitam danos à saúde decorrentes de um alimento (SANTOS, 2011). 
Considerando a importância da conformidade da rotulagem para a orientação do consumidor, objetivou-se com esse trabalho investigar a rotulagem de diferentes marcas de salgadinhos a base de milho comercializados na cidade de Pombal, Paraíba, de acordo e compará-las com as principais legislações vigentes.

\section{FUNDAMENTAÇÃO TEÓRICA}

Segundo a legislação brasileira, define-se rótulo como toda inscrição, legenda ou imagem, ou toda matéria descritiva ou gráfica, escrita, impressa, estampada, gravada, gravada em relevo ou litografada ou colada sobre a embalagem do alimento (ANVISA, 2002). De acordo com o Código de Proteção e Defesa do Consumidor, os rótulos atuam como um veículo de segurança alimentar ou nutricional, de modo que garantam a saúde pública e o direito humano à alimentação com qualidade (LOBANCO et al, 2009).

Com o ritmo de vida acelerado das pessoas, é comum a busca por alimentos de rápido e fácil consumo, ou seja, os que ofereçam uma maior praticidade ao consumidor. E os alimentos industrializados se encaixam nesse mercado, elevando de maneira expressiva o consumo de alimentos ricos em gorduras, sais e açucares (GARCIA; CARVALHO, 2011; ARAUJO, 2017).

A indústria de alimentos e também os órgãos responsáveis, buscam aplicações significativas, através de revisões de projetos e regulamentações adequadas relacionadas à rotulagem de alimentos, a fim de auxiliar os consumidores a fazer suas escolhas de maneira mais fácil e correta entre uma infinidade de produtos disponíveis no mercado, para que saiba o que se deseja consumir ou não na sua dieta (SHEN et al., 2018).

Ao orientar o consumidor sobre a qualidade e a quantidade dos constituintes nutricionais dos produtos, a rotulagem dos alimentos pode promover escolhas alimentares adequadas, desse modo é indispensável à fidedignidade das informações. Porem, falhas na legislação vigente no Brasil têm sido observadas, levando informações incorretas e causando confusão ao consumidor (CÂMARA et al., 2008; GARCIA et al., 2015).

A disponibilidade de informações corretas e de forma compreensível ao leitor, quanto a tabela nutricional, podem corrigir erros alimentares, levando a contribuição de maneira positiva de modo que promova a saúde e a redução do risco de doenças relacionadas à desnutrição e à alimentação inadequada na fase infantil (GARCIA et al., 2015).

Os salgadinhos estão entre os alimentos de maior preferência e de elevado consumo, principalmente pelas crianças. O consumo desenfreado desses tipos de alimentos causa preocupação, pois se sabe que esses alimentos possuem elevador teores de sódio e que o seu 
consumo de forma excessiva está associado ao aumento da pressão arterial (FEDALTO et al., 2011).

\section{METODOLOGIA}

A pesquisa descritiva foi realizada no comércio local da cidade de Pombal, Paraíba, Brasil, no primeiro semestre de 2017. Foram analisadas 7 diferentes marcas de salgadinhos a base de milho (peso líquido médio de 53,5 g) comercializadas em supermercados, mercadinhos e lanchonetes. As marcas analisadas foram as únicas encontradas nos estabelecimentos visitados.

As informações contidas nos rótulos foram coletadas por meio do auxílio de uma lista de verificação com 36 itens de múltipla escolha (opções sim, não e não se aplica) disponibilizado pela Agência Nacional de Vigilância Sanitária (ANVISA) na internet, tendo como base o Decreto-lei n 989 de 19691, RDC n 259 de 2002, RDC no 344 de 2002, RDC n 360 de 2003, RDC nº 123 de 2004 e a Lei ${ }^{\circ} \mathbf{1 0 . 6 7 4}$ de 2003, contendo questões referentes a: presença de corantes e aromatizantes naturais e artificiais; declarações superlativas e materiais de propaganda; alegações funcionais e/ou de saúde; representações gráficas falsas; componentes presentes/ausentes, adicionados aos alimentos e propriedades sensoriais; expressão "tipo" para vinhos e bebidas alcoólicas; produtos importado; informações obrigatórias; lista de ingredientes; uso de aditivos; identificação do fabricante e produto; lote e data de validade; indicação de preparo e instruções de uso; informação nutricional; expressão "contém glúten" ou "não contém glúten" e designação de produtos fortificados ou enriquecidos, conforme Tabela 1.

Todos os rótulos são de procedência nacional e possuem tamanhos e preços distintos. Os dados foram tabulados no Microsoft Excel® 2007 e analisados em número e percentual, por meio de gráficos. Por se tratar de uma pesquisa com alimentos o trabalho não precisou ser submetido ao Comitê de Ética em Pesquisa.

\section{RESULTADOS E DISCUSSÃO}

A Figura 1 apresenta os resultados de conformidade e não conformidade, dentro de cada marca avaliada. 
Figura 1 - Rótulos conformes, não conformes e não se aplica por marca. Fonte: Própria

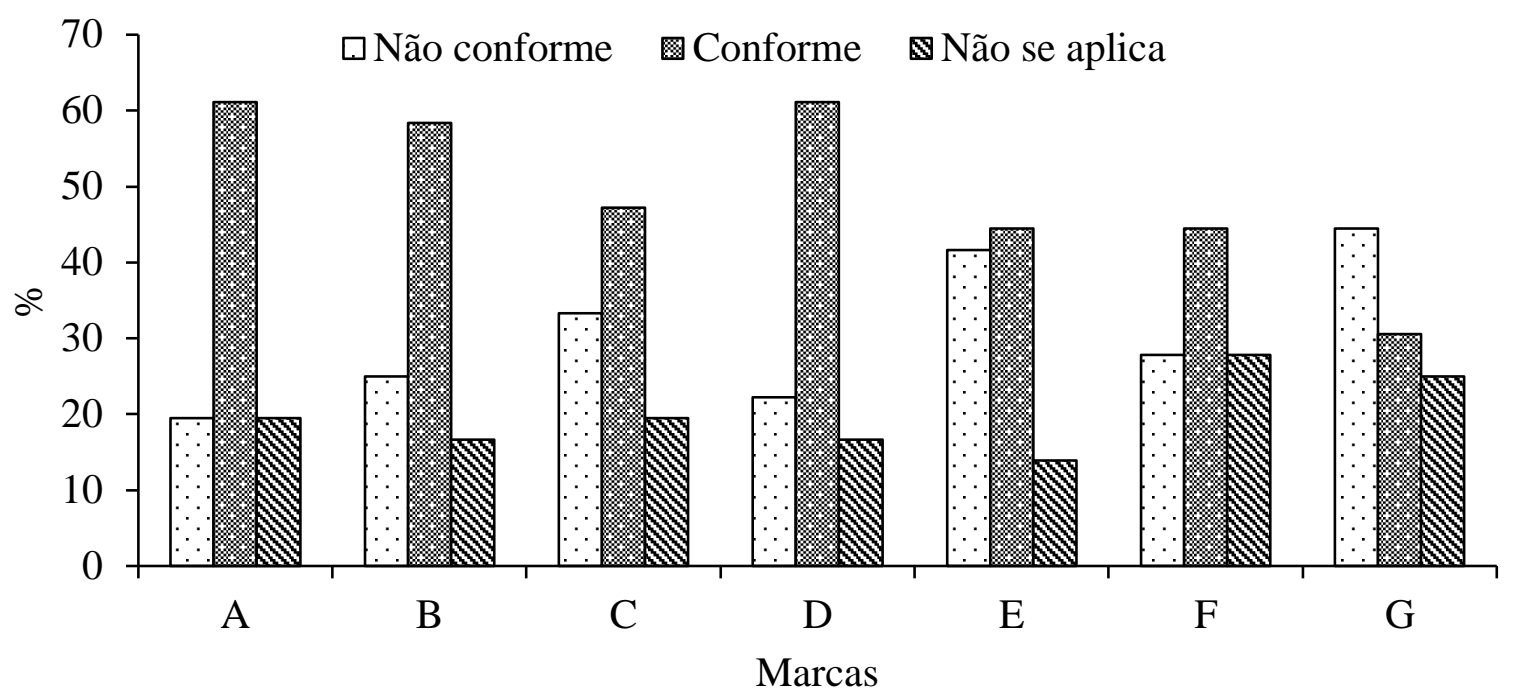

É possível observar que a maioria das marcas apresentaram grandes quantidades de conformidade, estando elas com valores variando de $30,55 \%(\mathrm{G})$ a $61,11 \%$ (A), apenas para a marca $\mathrm{G}$ os valores de não conformidades foram superiores aos conformes.

Existem elevadas inadequações presentes nos rótulos de alimentos, principalmente quanto às informações nutricionais apresentadas, mas essas inadequações resultam mais da falta de fiscalização do que da ausência de leis. É evidente a contribuição das normas e leis sobre rotulagem no Brasil, todavia, são necessárias ações mais eficazes para o seu cumprimento nas indústrias de alimentos (CÂMARA et al., 2008).

As marcas A e D apresentaram valores iguais quanto à conformidade dos rótulos, de $61,11 \%$. O mesmo aconteceu com as marcas E e F que também proporcionaram os mesmos valores, de 44,44\%. As marcas B e C apresentaram 58,33 e 47,22\% e conformidade, respectivamente.

$\mathrm{Na}$ Tabela 1 estão apresentados os resultados para conformidade (S), não conformidade (N) e não se aplica (NA) de cada parâmetro avaliado, de acordo com a adaptação da lista de verificação disponibilizada pela ANVISA na internet.

Tabela 1- Conformidade (S), não conformidade (N) e não se aplica (NA) de cada parâmetro avaliado.

$\begin{array}{lllllll}\text { A } & \text { B } & \text { C } & \text { D } & \text { E } & \text { F } & \text { G }\end{array}$

1. O produto contém corantes artificiais? Se sim, responder a $\mathbf{N}$ questão 2.

\begin{tabular}{|c|c|c|c|c|c|c|}
\hline $\mathbf{N}$ & $\mathbf{N}$ & $S$ & $\mathbf{S}$ & $\mathbf{N}$ & $\mathbf{N}$ & $\begin{array}{l}\text { Art. } 13 \text { do } \\
\text { DL } 986 / 69\end{array}$ \\
\hline
\end{tabular}


2. O rótulo do produto apresenta a declaração "Colorido Artificialmente"?

N $\quad \mathbf{N}$

3. O produto é adicionado de essências naturais ou artificiais? Se sim, responder as questões 4 e 5.

4. O rótulo apresenta, no painel principal, a declaração "Contém Aromatizante" e, caso utilize aroma artificial, apresenta a declaração "Aromatizado Artificialmente"?

5. Caso utilize essências naturais, o rótulo apresenta, no painel principal, a declaração "Sabor de..." e "Contém Aromatizante"?

6. O rótulo apresenta declarações superlativas de qualidade em discordância com o RT específico?

7. Os materiais de propaganda ou qualquer outro veículo de divulgação estão de acordo com os dizeres de rotulagem?

8. O rótulo traz indicações medicamentosas ou terapêuticas?

NA $\quad \mathbf{N}$

$\mathbf{N}$

$\mathbf{N}$

$\mathbf{N}$

Art. 56 do DL $986 / 69$ Item 3.1.e e $f$ da RDC 259/2002

9. O rótulo aconselha o consumo do produto como estimulante, para melhorar a saúde ou para prevenir doenças?

NA

$\mathbf{N}$

$\mathbf{N}$

$\mathbf{N}$

$\mathbf{N}$

$\mathbf{N}$

$\mathbf{N}$

Item 3.1.g

da RDC 259/2002

10. O rótulo apresenta vocábulos, sinais, denominações, símbolos, emblemas ou outras representações gráficas que possam tornar a informação falsa, incorreta, insuficiente, ou que possa induzir o consumidor a equívoco, erro, confusão ou engano, em relação à natureza, composição, procedência, tipo, qualidade, quantidade, validade, ou forma de uso do alimento?

11. O rótulo atribui efeitos ou propriedades que o produto não possua ou não possam ser demonstradas?

$\begin{array}{lllllllll}\mathbf{N} & \mathbf{S} & \mathbf{N} & \mathbf{N} & \mathbf{S} & \mathbf{N} & \mathbf{N} & \begin{array}{l}\text { Item } \\ \text { da } \\ 259 / 02\end{array}\end{array}$

N $\mathbf{N}$

$\mathbf{N}$

$\mathbf{N}$

$\mathbf{N}$

$\begin{array}{ll}\text { Item } & 3.1 . b \\ \text { da } & \text { RDC } \\ 259 / 02 & \end{array}$ 
12. O rótulo destaca a presença ou ausência de componentes que sejam intrínsecos ou próprios de alimentos de igual natureza?

13. O rótulo ressalta a presença de componentes que sejam adicionados como ingredientes em todos os alimentos similares?

14. Os rótulos dos alimentos fabricados com propriedades sensoriais semelhantes ou parecidas com aquelas típicas de lugares geográficos reconhecidos apresentam na denominação a expressão "tipo" com letras de igual tamanho, realce $\mathrm{e}$ visibilidade?

15. O rótulo utiliza a expressão "tipo" para denominar vinhos e bebidas alcoólicas? Obs: não é permitido.

16. O rótulo de produtos importados traz etiqueta com as informações obrigatórias em português?

17. A informação obrigatória está em português com caracteres de tamanho, realce e visibilidade adequados?

18. O rótulo traz todas as informações obrigatórias: designação, lista de ingredientes, conteúdo líquido, identificação da origem, razão social, lote, prazo de validade e instruções de preparo, se necessário? Obs.: quando a superfície do painel principal for inferior a $10 \mathrm{~cm}^{3}$ pode constar apenas a designação e a marca.

19. A designação do produto e o conteúdo líquido estão declarados no painel principal do rótulo?

20. O tamanho das letras e números da informação obrigatória tem no mínimo $1 \mathrm{~mm}$ ? 21. A lista de ingredientes é precedida da expressão "ingredientes" ou "ingr.:"? Para alimentos com com ingrediente único, não há necessidade de declarar a lista de ingrediente (ex. açúcar, farinha, erva-mate, vinho, etc.)

22. A lista de ingredientes está em ordem decrescente da respectiva proporção?

23. O ingrediente composto contido em um alimento está declarado como tal na lista de ingredientes, acompanhado imediatamente de uma lista entre
S N

$\mathbf{N}$

$\mathbf{N}$

$\mathbf{N}$

$\mathbf{N}$

Item 3.1.c

da RDC $259 / 02$

Item 3.1.d

da RDC $259 / 02$

Item $3.3 \mathrm{da}$

RDC

$259 / 02$

NA NA

NA

NA

NA

NA

NA

Art. 1 da

Res RDC

123/04

Item 3.4 da

RDC

259/02

Item 4 da

RDC

259/02

Itens 5 e 9.1

da RDC $259 / 02$

Itens 6.3 e 8

da RDC n.

$259 / 02$

Item 8.2 da

RDC $\mathrm{n}^{\circ}$

259/02

Itens $6.2 .1 \mathrm{e}$

6.2.2 da

RDC

259/02

Item 6.2.2.a

da RDC

259/02

Itens 6.2.2.b

e 6.2.2.c da

RDC

259/02 
parênteses de seus componentes? Obs.: este item não se aplica para os ingredientes compostos estabelecidos na norma CODEX e que representam $25 \%$ do produto acabado (exceção aditivos)

24. O produto que contém água em sua composição traz este componente na lista de ingredientes? Obs.: O produto que contém salmouras, xaropes, $\mathbf{N}$ caldas, molhos ou outros similares pode declarar estes ingredientes como tais na lista de ingredientes.

25. A função principal do aditivo, o nome completo ou número INS estão declarados na lista de ingredientes? Obs.: Os aromas podem ser declarados como aromas/aromatizantes.

26. Consta no rótulo a razão social, o endereço completo, país de origem, município, número de registro (quando for o caso) e CNPJ?

27. Consta identificação da origem para produto importado ${ }^{6}$ ? "Indústria...", "Produto...",

"Fabricado em..."

28. O lote pode ser declarado por meio de código precedido pela letra "L" ou pela data de fabricação/embalagem/prazo de validade sempre que constar no mínimo o dia e o mês ou o mês e o ano.

Há identificação do lote, conforme acima?

29. O prazo de validade deve ser apresentado no mínimo Dia/Mês para produtos com prazos de validade inferior a 3 meses e Mês/Ano para produtos com prazo de validade superior a 3 meses, precedido de uma das seguintes expressões: "consumir antes de...", "válido até...", "val:...", "validade", "vence...", "vencimento...", "vto:...", "venc:...", "consumir preferencialmente antes de...". O prazo de validade do produto está adequado?

30. As expressões da questão 29 estão acompanhadas do prazo de validade ou uma indicação clara do local onde consta o prazo de validade ou de impressão através de perfurações ou marcas indeléveis do dia, mês e ano? 31. Consta da rotulagem a descrição dos cuidados de armazenamento e conservação para produto que necessita

Item $\quad 6.2 .4$

da RDC $259 / 02$

Item $\quad 6.4 .1$

da RDC n. ${ }^{\circ}$ $259 / 02$

Item 6.4.2. da RDC n. ${ }^{\circ}$ $259 / 02$

Item 6.5 da $\mathrm{RDC} \quad \mathrm{n} .^{\circ}$ 259/02
Item $6.6 \mathrm{da}$ RDC n. $259 / 02$

Item 6.6.1.d da RDC ${ }^{\circ}$ $259 / 02$

Item 6.6.2. da RDC n. ${ }^{\circ}$ $259 / 02$

\footnotetext{
${ }^{6}$ Uma vez que para o produto nacional já deve constar nome ou razão social e endereço completos da empresa fabricante.
} 
cuidado especial de conservação? 32. Consta da rotulagem indicação de preparo e instruções de uso do produto, quando for o caso?

33. Há informação nutricional na rotulagem do produto (observar a adequação da legislação)?

34. Há a expressão "Contém Glúten" ou "Não contém Glúten?

35. As farinhas de trigo e de milho são designadas com o nome convencional do produto acrescido de uma das expressões: fortificada ou enriquecida ou rica com ferro e ácido fólico?

36.As farinhas de trigo e de milho usadas como ingredientes são declaradas na lista de ingredientes do produto com uma das seguintes expressões: farinha de trigo ou de milho fortificada ou enriquecida ou rica com ferro e ácido fólico?

\begin{tabular}{|c|c|c|c|c|c|c|c|}
\hline $\mathbf{S}$ & NA & NA & NA & NA & NA & NA & $\begin{array}{ll}\text { Item } 6.7 \text { da } \\
\text { RDC } & \text { n. } \\
259 / 02 & \end{array}$ \\
\hline $\mathbf{S}$ & $\mathbf{S}$ & $\mathbf{S}$ & $\mathbf{S}$ & $\mathbf{N}$ & $\mathbf{S}$ & $\mathbf{S}$ & $\begin{array}{l}\text { Resoluções } \\
\text { RDC n. }{ }^{\circ} \\
39 \text { e } 40 / 01 \\
\text { ou RDC n } \\
360 / 2003 .\end{array}$ \\
\hline $\mathbf{S}$ & $\mathbf{S}$ & $\mathbf{S}$ & $\mathbf{S}$ & $\mathbf{S}$ & $\mathbf{S}$ & $\mathbf{S}$ & $\begin{array}{l}\text { Lei n. } .^{\circ} \\
10.674 / 200 \\
3\end{array}$ \\
\hline $\mathbf{S}$ & $\mathbf{S}$ & $\mathbf{S}$ & $\mathbf{S}$ & $\mathbf{N}$ & NA & $\mathbf{N}$ & $\begin{array}{l}\text { Item } 5.1 \text { da } \\
\text { RDC n } \mathrm{n}^{\circ} \text {. } \\
344 / 2002\end{array}$ \\
\hline $\mathbf{S}$ & $\mathbf{S}$ & $\mathbf{S}$ & $\mathbf{S}$ & $\mathbf{N}$ & NA & $\mathbf{N}$ & $\begin{array}{l}\text { Item } 5.2 \text { da } \\
\text { RDC n } \text { n. }^{\circ} \text {. } \\
344 / 2002\end{array}$ \\
\hline
\end{tabular}

Observa-se na Figura 2 a apresentação das itens conformes, não conformes e não se aplica de cada categoria de itens compilados e obtidos da Tabela 1.

Figura 2 - Categoria dos itens conforme, não conforme e não se aplica. Fonte: Própria

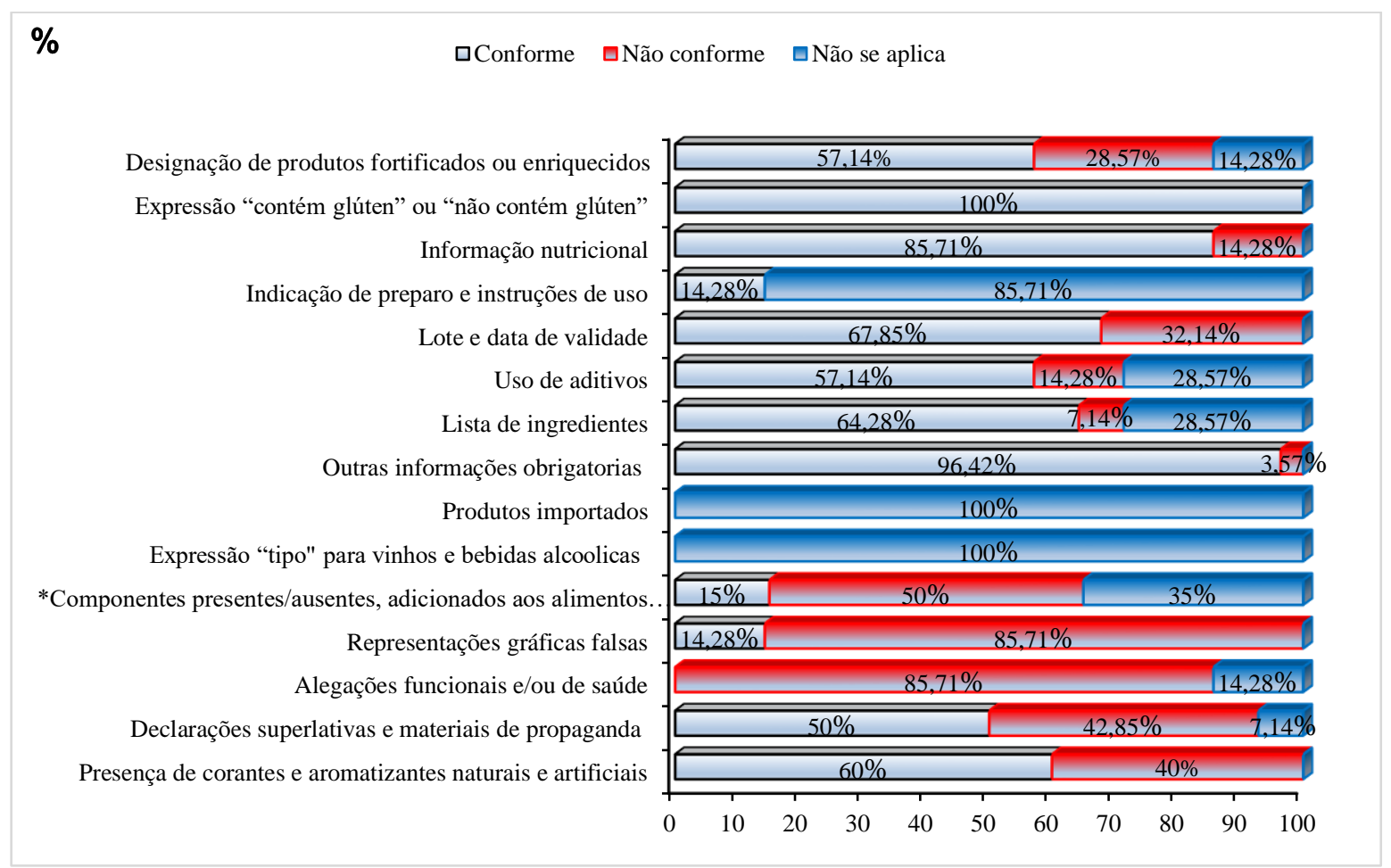

*Componentes presentes/ausentes, adicionados aos alimentos e propriedades sensoriais

Na categoria de Designação de produtos fortificados ou enriquecidos o percentual de 
conformidades foi superior do que os de não conformidades e não se aplica, expressando valores de 57,14, 28,57 e 14,28\% respectivamente. Para a categoria "Contém Glúten" ou "Não contém Glúten" continha apenas um item na qual 100\% das marcas apresentaram conformidades quanto a apresentação no rótulo.

Dentre os itens analisados para a categoria de Informação nutricional, 85,71\% estavam conformes, segundo a legislação vigente. A Indicação de preparo e instruções de uso apresentam maiores valores para a opção não se aplica, por se tratar de produtos que são prontos para o consumo, entretanto em 14,28\% dos itens analisou-se a presença de instruções de uso.

Para a ategoria Lote e data de validade, apesar de ser um dos itens mais importantes do rótulo, ainda foram encontrados valores de 32,14\% de não conformidade para este item, isso pode ser explicado pela falta de atenção do responsável técnico ou pela má qualidade da tinta utilizada na litografia, sendo essa removida facilmente.

Nas categorias Uso de aditivos, Lista de ingredientes e Outras informações obrigatorias a maioria dos itens apresentaram conformidades. Para Produtos importados todos os itens foram não se aplicam, pois todas as marcas analisadas eram de origem brasileira, não havendo necessidade de tradução. Para Componentes presentes/ausentes, adicionados aos alimentos e propriedades sensoriais, 50\% dos itens form não conforme por não apresentar no rótulo, $35 \%$ foram conformes e $15 \%$ não se aplica a um determinado item.

Representações gráficas falsas e alegações funcionais e/ou de saúde não estavam presentes em $85,71 \%$ de ambos os itens, em apenas $14,28 \%$ foi analisado a presença dessas alegações. De acordo com Câmara et al. (2008), 43\% dos consumidores de alimentos no Brasil, buscam nas embalagens informações sobre os benefícios para a saúde no ato da compra dos alimentos. Os entrevistados por esses autores acreditam na capacidade de prevenção e controle que a alimentação pode exercer sobre doenças, tais como o câncer, as doenças do coração, a hipertensão arterial e a obesidade.

Em $60 \%$ dos rótulos foi verificado a presença de Corantes e aromatizantes, tanto naturais como sintéticos, em $40 \%$ não foi verificado. Rodrigues et al. (2012), observaram que todos os produtos analisados em seu estudo sobre a presença de edulcorantes nos rótulos de alimentos encontravam-se dentro dos padrões exigidos pela legislação, verificado assim, que a indústria de alimentos tem buscado repassar informações mais claras ao consumidor e se adequando aos padrões exigidos pelas legislações no país. 


\section{CONCLUSÕES}

Este trabalho permitiu identificar dados de conformidade da rotulagem de produtos alimenticios frente as legislações vigentes em vigor. Dentre as sete marcas analisadas destacase as marcas A, B, C, D, E e F por apresentarem valores de conformidades superiores as não conformidades, verificando que apenas a marca $\mathrm{G}$ apresentou maiores valores não conformes, caracterizando um resultado favoravel, pois mais de $90 \%$ das marcas atendiam as legislações vigentes. No entanto, ainda se faz necessario um comprometimento das indústrias frente as rótulagens impostas para cada produto, além de uma intensa fiscalização dos orgãos responsaveis para que a mesma seja realizada de acordo com as determinações legais.

\section{REFERÊNCIAS}

ARAUJO, W. D. R. Importância, estrutura e legislação da rotulagem geral e nutricional de alimentos industrializados no Brasil. Revista Acadêmica Conecta FASF, v. 2, n. 1, p. 35-50, 2017.

BENDINO, N. I.; POPOLIM, W. D.; OLIVEIRA, C. R. A. Avaliação do Conhecimento e Dificuldades de Consumidores Frequentadores de Supermercado Convencional em Relação à Rotulagem de Alimentos e Informação Nutricional. Revista do Instituto de Ciências da Saúde, v.30, n.3, p:261- 265, 2012.

BRASIL. Ministério da Saúde. Agência Nacional de Vigilância Sanitária, ANVISA. Legislações sobre rotulagem de alimentos. Disponível em: < http://portal.anvisa.gov.br/registros-e-autorizacoes/alimentos/produtos/rotulagem>. Acesso em: 01 de out. 2018.

CÂMARA, M. C. C.; MARinhO, C. L. C.; GUILAM, M. C.; BRAGA, A. M. C. B. A Produção Acadêmica sobre Rotulagem de Alimentos no Brasil. Revista Panamericada de Salud Pública/Pan American Journal of Public Health, v. 23 n.1, p: 52-58, 2008.

FEDALTO, M. B.; OLIVEIRA, J.; STOFELLA, N. C. F.; BALBI, M. E. determinação do teor de sal em salgadinhos de milho e possíveis consequências na alimentação infantil. Visão Acadêmica, v.12, n.1, 2011.

GARCIA, M. R.; VIEITES, R.; DAIUTO, E. Avaliação nutricional e conformidade da rotulagem de "petiscos" consumidos por crianças à legislação brasileira. Energia na Agricultura, v. 30, n.1, p.80-86, 2015.

GARCIA, P. P. C.; CARVALHO, L. P. da S. Análise da rotulagem nutricional de alimentos diet e light. Ensaios e Ciência, São Paulo, v. 15, n. 4, p. 89-103, 2011.

GUIA ALIMENTAR PARA A POPULAÇÃO BRASILEIRA. Ministério da saúde. Secretaria de atenção à saúde. Departamento de atenção básica. Coordenação geral da política de alimentação e nutrição. Brasília/ DF 2006. Disponível em: <http://dtr2001.saude.gov.br/editora/produtos/livros/pdf/05_11091_M.pdf>. Acesso em: 01 out. 2018. 
LOBANCO, C. M.; VEDOVATO, G. M.; CANO, C. B.; BASTOS, D. H. M. Fidedignidade de rótulos de alimentos comercializados no município de São Paulo, SP. Revista Saúde Pública, São Paulo, v. 3, n. 43, p. 499-505, 2009.

PEIXOTO, L. O.; AZEVEDO, C. V.; FREITAS, B. K. S. Avaliação do Teor de Sódio, das Porções e das Medidas Caseiras em Salgadinhos Industrializados. Revista de Nutrição e Vigilância em Saúde, v.1, n.2, p: 11-15, 2014.

RODRIGUES, F. R.; SALDANHA, T.; BARBOSA, M. I. M. J. Avaliação da presença de edulcorantes nos rótulos de produtos alimentícios. Acta Tecnológica, v. 7, n. 1, p. 38-43, 2012.

SANTOS, C. M. B. Segurança alimentar e Rotulagem de Alimentos sob a Perspectiva do Código do Consumidor Brasileiro. Revista Percurso Acadêmico, v. 1, n. 1, p. 327-346, 2011.

SHEN, M.; SHI, L.; GAO, Z. Beyond the food label itself: How does color affect attention to information on food labels and preference for food attributes?. Food Quality and Preference. v.64, p. 47-55, 2018. 